\title{
Different architectures of collagen fibrils enforce different fibrillogenesis mechanisms
}

\author{
Mario Raspanti \\ Laboratory of Human Morphology, Insubria University 71 Via Monte Generoso, Varese, Italy. \\ Email: mario.raspanti@uninsubria.it
}

Received 25 October 2010; revised 29 October 2010; accepted 1 November 2010

\begin{abstract}
According to current knowledge on collagen fibrillogenesis, collagen fibrils are formed by a cooperative process involving lateral fusion of small protofibrils. Almost all the experimental research, however, was carried out on tendon collagen, whose fibrils are characterized by approximately straight subfibrils. By contrast, in most tissues the collagen fibril subunits follow a helical course in which geometrical constraints prevent lateral fusions, thereby implying a different mechanism where collagen fibrils grow by addition of individual microfibrils rather than by lateral fusion of pre-assembled subfibrils. The process at the origin of these fibrils may provide a simple, automatic explanation for the remarkable uniformity in fibrils size observed in most tissues without requiring the intervention of unknown mechanisms of diameter control. Other mechanisms of growth control remain indispensable to terminate the fibrillogenesis process in tendons and ligaments.
\end{abstract}

Keywords: Fibrillogenesis; Microfibrils; Collagen Fibril Substructure

\section{INTRODUCTION}

Fibrillar collagens account for approximately a third of the total body proteins. Their molecules, a coiled coil of three individual polypeptide chains, ultimately form discrete fibrils of indeterminate length and widely variable diameter [1].

The diameter of collagen fibrils plays a central role in the functional behaviour of the diverse connective tissues $[2,3]$, but the mechanisms controlling fibril size remain unsettled. A number of different molecules and factors have been shown to influence the collagen fibril growth, including the relative content of collagen III [4] and $\mathrm{V}[5]$, the terminal globular domains of the collagen molecule (especially the N-terminal propeptide [6]), cross-links [7], fibril-associated non-helical collagens (or FACITs), COMP [8], small proteoglycans such as decorin [9], fibromodulin [10], lumican [11] and biglican [12], but also tenascin [13], perlecan [14], matrilin [15] and thrombospondin [16]. Although there is some evidence for most of these factors, none of them is by itself entirely consistent with all the experimental observations. In particular none was able to explain why in most tissues the fibril growth is very effectively controlled so that the collagen fibrils size is highly uniform and distributed along a narrow Gaussian curve, while in other, and often adjoining, tissues the collagen fibrils are large and inhomogeneous with a distinctive multimodal distribution. The difference between these two classes is so great that it may seem not unreasonable to hypothesize that two entirely different mechanisms are at work in different tissues.

It is important to bear in mind that these two classes of fibrils represent two mutually exclusive subfibrillar architectures [17-19]. Historically, research on fibril architecture and fibrillogenesis has been carried out almost exclusively on the large variable fibrils of tendons, whose subfibrils run almost parallel to the fibril axis. These fibrils come into being by means of a cooperative mechanism involving the lateral fusion of smaller protofibrils [20]: the process has been directly observed in vivo, and in vitro even mature fibrils of type I collagen retain the ability to fuse laterally in thick clumps unless fusion is inhibited by small proteoglycans [21].

Much less attention has been paid to the smaller unimodal fibrils. Corneal fibrils have been painstakingly demonstrated to be made of discrete subunits, corresponding in size to the microfibrils of Smith [22] and laid out in concentric layers $[23,24]$ where they wind in a right-handed helix. In each layer the microfibrils wind at a constant angle of 15 to 17 degrees with respect to the fibril axis, the precise measurement depending on the technique used. As a consequence, the axial D-period is reduced from the usual $67 \mathrm{~nm}$ to approximately $64 \mathrm{~nm}$ 
$\left(64 \approx 67 \cdot \cos \left(17^{\circ}\right)\right)[25]$, and the tilt-caused shear brings adjoining microfibrils in such a position to allow the formation of a peculiar covalent cross-link involving a histidine residue [26]. The diameter of these "helical" fibrils varies from tissue to tissue but remains extremely uniform in each location. Other tissues with small heterogeneous fibrils (such as blood vessels and nerve sheaths) have been less studied, but the facts that they all share an identical winding angle, the same shortened D-period and an equally narrow diameter distribution are strongly suggestive of an identical substructure. This subfibrillar architecture, defined as a constant angle helix and originally entertained in 1989 [19], is not easy to visualize: the pitch of the helix that each subfibril describes around the fibril axis is $2 \pi r \cdot \tan (\pi / 2-\alpha)$ (where $\alpha$ is the angle with respect to the fibril axis) and therefore, all other factors being constant, it is variable with the radius.

On the other hand, this design automatically guarantees that all the microfibrils have an identical axially-projected length of $64 \mathrm{~nm}$ (an essential condition for the distinctive banding pattern of collagen to appear). The only alternative model, the constant pitch helix, requires axial subunits to be compressed and peripheral ones stretched in order to be consistent with ultrastructural data [19]. Moreover, because of its layered structure, the constant angle helix has the additional advantage of being consistent with the discrete distribution of diameter values reported in some tissues [2].

This structure has another simple but critical consequence which, to the best of our knowledge, has not been noticed so far: such helical fibrils cannot possibly undergo lateral fusion since the adjoining microfibrils of two parallel fibrils always wind in opposite directions (Figure 1). This, of course, also holds true for antiparallel fibrils, which would be unable to merge anyway. It is an everyday observation that wire ropes are made of strands that never merge: for a helically-wound fibril to merge with another similar fibril, it would have to be entirely unwound and rewound, a process made unlikely by the substantial variation of free energy it implies.

In other words, while the large heterogenous size of tendon collagen fibrils may emerge as the result of a random lateral fusion of protofibrils taking place during the first phases of fibrillogenesis (until this process is inhibited by fibril-bound proteogycans and/or by some other mechanism), the slender fibrils of cornea, blood vessels and sheaths must simply precipitate from a supersaturated solution of collagen molecules. Under these conditions each growing fibril competes with its neighbours for the available subunits until all these have been depleted. The extracellular environment being the same for all forming fibrils in a given location, they all

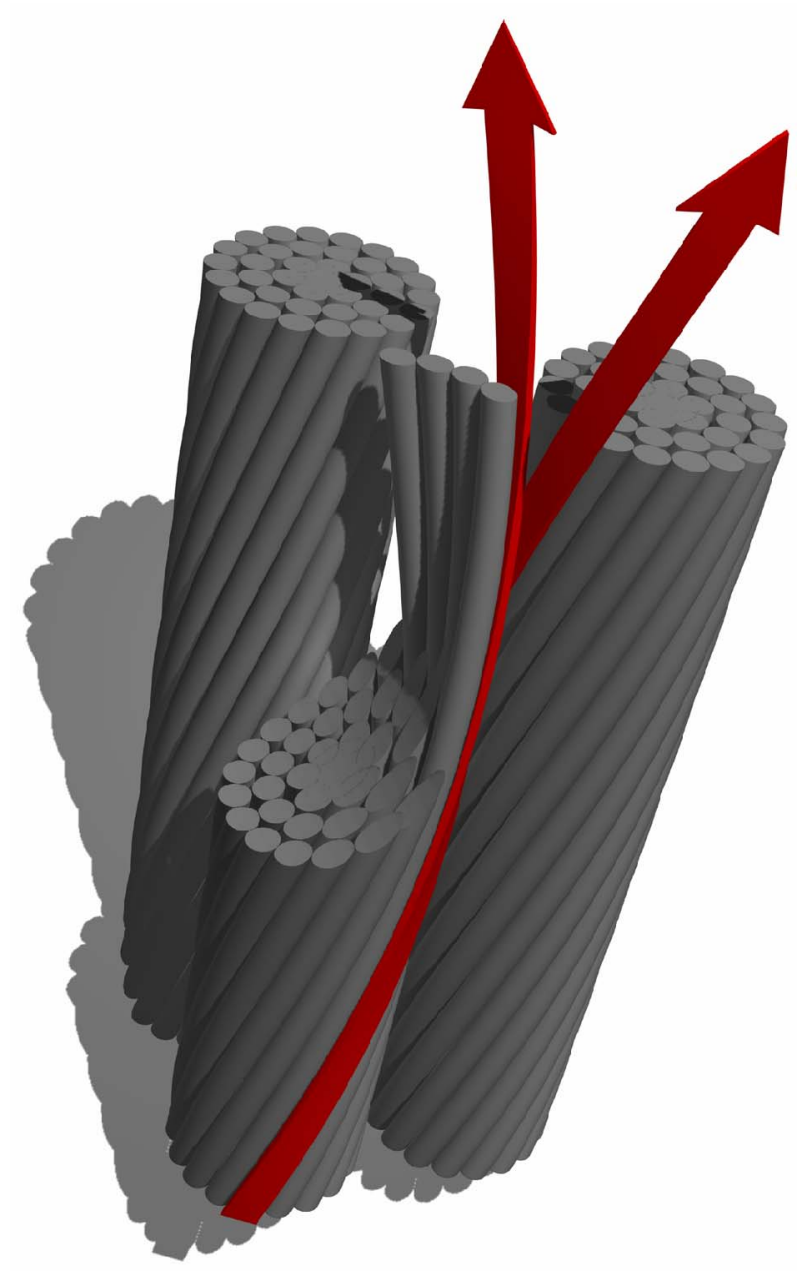

Figure 1. The adjoining microfibrils of two parallel fibrils run in opposite directions (red arrows) preventing any lateral fusion. Model developed on Alias|Wavefront Maya 6.0.1 running under IRIX on a Silicon Graphics Fuel R14000/600.

end up of a similar size without requiring (at least in principle) any external limiting factor. Again, this phenomenon is readily observed in everyday life: for instance in snowflakes or hailstones which, albeit variable from place to place, tend to have the same size in the same location.

\section{MODEL DEVELOPMENT}

A simple program was developed to test this hypothesis. The program essentially tries to obtain a graphical simulation of the cross-section of a bundle of cylindrical fibrils. Each individual pixel represents a 4-nm microfibril; the program typically manages a $500 \times 500$ pixel arena, corresponding to a $2000 \times 2000 \mathrm{~nm}$ field, which is enough to create a few hundred fibrils, which in turn can represent a significant sample.

The program can be outlined as follows:

1) A new microfibril is created in a random position 
$(x, y)$.

2) If no fibril lies within a given "capture distance" (a user-defined value set by the Field parameter - see Figure 2), then the microfibril itself becomes a new fibril; else the microfibril coalesces with its nearest fibril, whose radius is recomputed accordingly.

3) A test is made to check if the fibril, which has now grown larger, collides with any other fibril. If a collision is found:

4) If fibril merging is enabled (via the Merge switch) then the two fibrils merge into a new one, whose radius and position are recomputed; else the colliding fibril is simply displaced.

5) If fibril merging is enabled, a random function (whose probability increases with the fibril size) can block the fibril, preventing other subsequent fusions.

6) Point (3) is recursively repeated until no further collisions are found.

The whole process is iterated up to the desired volume fraction (set by the Fraction parameter).

As in any simulation, a few arbitrary assumptions had to be made:

1) In the real world the capture distance is likely to depend on a complex interplay of several factors which are impossible to quantify precisely. One of them is the creation rate of the microfibrils (if the rate is low enough there is more time for the Brownian motion to bring new microfibrils into contact with a preexisting fibril). In software this parameter was empirically set to a fraction of a collagen molecule length.

2) The volume fraction of collagen is also variable from tissue to tissue. In our simulation this parameter is not really important, since the fibrils in excess simply exit the simulation arena and are lost, so it was set to $100 \%$.

3) The termination condition of point (5) was introduced to prevent fusion-enabled fibrils merging into a single huge unit, that is precisely what happens in vitro when no control factors are present [21].

\section{RESULTS}

Even under these relatively crude conditions the pictures show a clear similarity with the actual micrographs. If the lateral fusion is allowed then the process leads to the appearance of widely heterogenous aggregates (Figure 2) quite similar to the fibrils found in tendons and ligaments.

If fibril fusion is precluded, the simulation yields a field of remarkably uniform fibrils (Figure 3). In this case the process even mimics the apparent alignment of fibrils in rows, often visible in electron micrographs (compare these pictures with Figure 5 of Ref. [1]).

It must be stressed that the software is intended to just

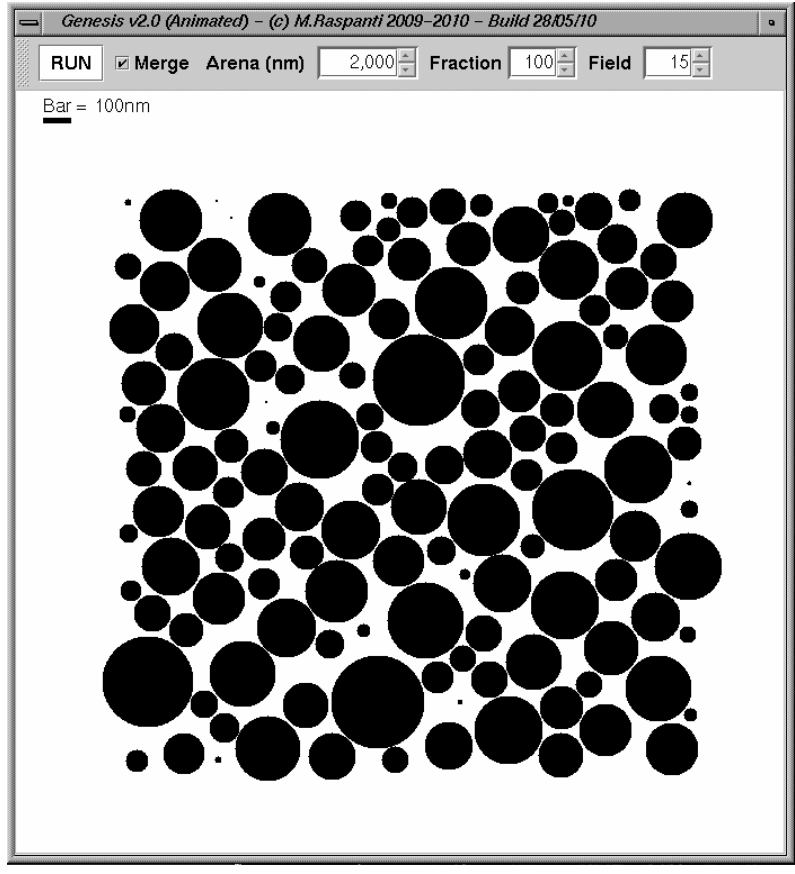

Figure 2. The graphical output of the fibrillogenesis simulation program. When fibril fusion is enabled, the microfibrils form fibrils with a widely variable distribution of diameters, ranging from $4 \mathrm{~nm}$ to over $450 \mathrm{~nm}$ as observed in tendons and ligaments.

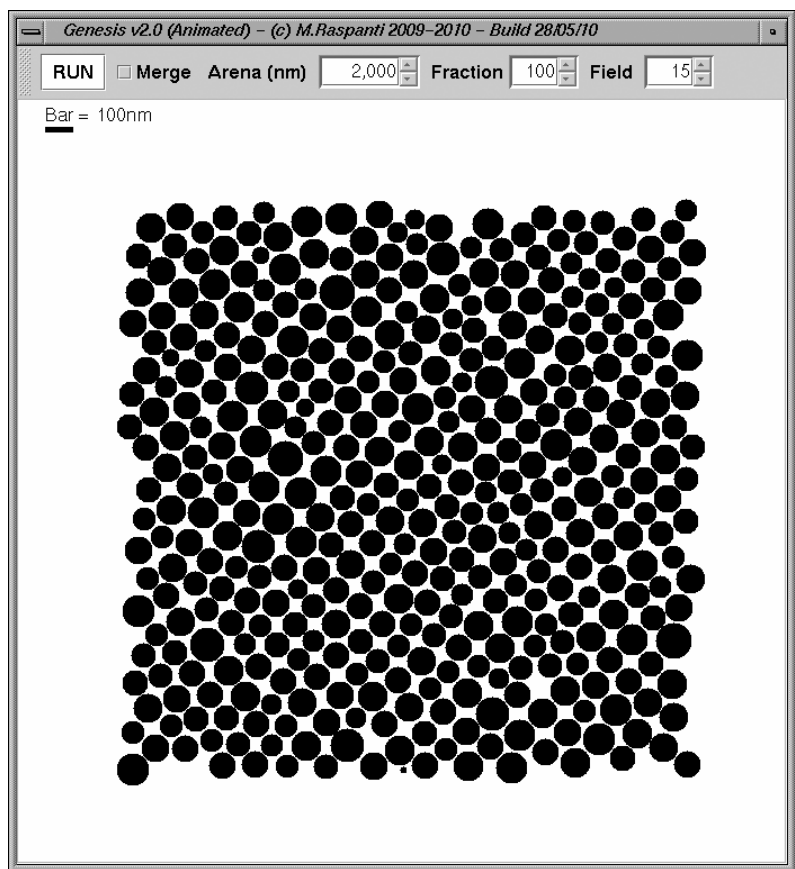

Figure 3. When the lateral fusion is disabled, all other parameters remaining constant, the result is a field of remarkably uniform fibrils with a diameter centered around $100 \mathrm{~nm}$ in a narrow distribution. The simulation software was written in standard Java 2 and runs on the same workstation as Figure 1. 

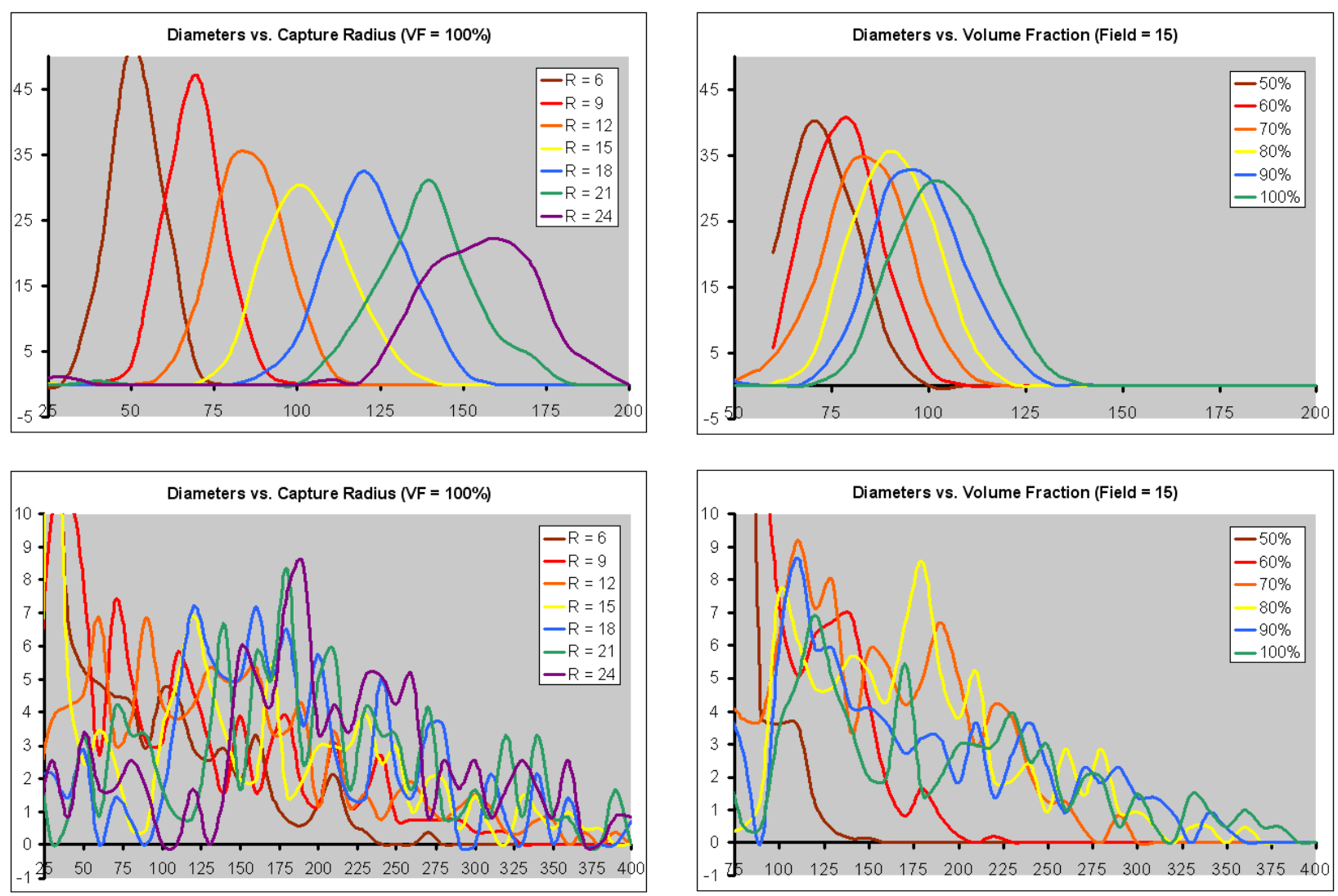

Figure 4. Exploring the parameter space in our simulation changes the average diameters but does not alter their distribution. The two panels above show the diameter distribution (in $\mathrm{nm}$ ) obtained when the lateral fusion is not allowed: changing the two user-defined parameters always produces a family of Gaussian curves, centered on different values but with a similar shape. The two panels below depict the multi-peak distribution obtained under the same conditions if the lateral fusion is enabled.

reveal a general trend, and does not claim to be a comprehensive reconstruction of the fibrillogenesis process. It is noteworthy, however, that varying all the parameters influenced the average diameter of fibrils but did not alter the diameter distribution: always a smooth Gaussian in one case, a multi-peak range in the other (Figure 4).

\section{DISCUSSION}

Our results suggest that the remarkable uniformity in size that collagen fibrils show in some tissues does not require the introduction of some unknown, stringent mechanism of diameter control, but emerges spontaneously as the result of a simple structural constraint. The most effective mechanism of growth control is one that does not depend on external interventions: the helical substructure of these fibrils is both a necessary and a sufficient condition for their diameter uniformity.

These results do not imply than propeptides or proteoglycans or other molecules have no part in limiting the lateral accretion of the fibrils; quite the opposite. The role, for instance, of SLRPs in this process is undeniable
[21], and even in our simulation a termination condition had to be introduced if lateral fusion was allowed. Rather, these results imply that the absence of external growth-limiting factors may affect different fibrils (and hence different tissues) in different ways. In tissues such as tendons whose fibrils have straight microfibrils, the proteoglycans (and/or propeptides or other molecules) are necessary to terminate lateral fibril aggregation whereas in tissues such as cornea, nerve sheaths and interstitial collagen of parenchymatous organs whose fibrils have helical microfibrils they are not. Therefore a DCN -/- organism, for instance, can be expected to show fibrillar alterations in tendons but not in cornea. And this is exactly what is found in experimental observations [9].

For historical and technical reasons connective tissue research is so deeply entrenched in the study of tendon and bone that the mere existence of fibrils with helical subunits took many years to emerge. Although independently confirmed by freeze-etching [17], cross-links analysis [26], SEM and AFM [27], electron tomography [23] and X-ray diffraction [24], the evidence has yet to 
find its way into histology textbooks. We must take care not to repeat the original sin of studying only tendons, and then ascribe what we found here to all other tissues.

Fibrils with helical subfibrils are the most common among our different tissues. Our data indicate that the current knowledge on the fibrillogenesis process, all gained on tendons, does not apply to these fibrils, and that new research is required.

The next primary objective will be the identification of the molecular switch controlling the initiation of one or the other subfibrillar architecture, and which has so far eluded detection. Fibrillogenesis is a delicate process which can be influenced by myriad causes, some of which do not even remain to make up the final product.

Perhaps the most promising candidate appears now type $\mathrm{V}$ collagen. Almost since its discovery the type $\mathrm{V}$ has associated with the control of fibril diameter [28], and more recently it seems to be essential for fibril formation in vivo: homozygote engineered organisms lacking type $\mathrm{V}$ die at embryonic day 10 of cardiovascular failure, while heterozygotes are viable but lack about half their collagen fibrils [29].

It seems therefore not unreasonable to hypothesize that type $\mathrm{V}$ collagen is involved in the formation of slender, uniform fibrils in cornea, blood vessels and sheaths, but obviously not in tendon and ligaments where it appears only in traces. Further research is underway.

\section{REFERENCES}

[1] Ottani, V., Martini, D., Franchi, M., Ruggeri, A. and Raspanti, M. (2002) Hierarchical structures in fibrillar collagens. Micron, 33, 587-596.

[2] Parry, D.A.D. and Craig, A.S. (1984) Growth and development of collagen fibrils in connective tissue. In: Ruggeri, A. and Motta, P.M., Eds., Ultrastructure of the Connective Tissue Matrix, Martinus Nijhoff, Hague, 36-64.

[3] Ottani, V., Raspanti, M. and Ruggeri, A. (2001) Collagen structure and functional implications. Micron, 32, 251-260.

[4] Birk, D.E., Zycband, E.I., Woodruff, S., Winkelmann, D.A. and Trelstad, R.L. (1997) Collagen fibrillogenesis in situ: fibril segments become long fibrils as the developing tendons matures. Developmental Dynamics, 208, 291-298.

[5] Chanut-Delalande, H., Fichard, A., Bernocco, S., Garrone, R., Hulmes, D.J.S. and Ruggiero, F. (2001) Control of heterotypic fibril formation by collagen $\mathrm{V}$ is determined by chain stoichiometry. Journal of Biological Chemistry, 276, 24352-24359.

[6] Bornstein, P. (2002) The NH(2)-terminal propeptides of fibrillar collagens: highly conserved domains with poorly understood functions. Matrix Biology, 21, 217-226.

[7] Eyre, D.R., Weis, M.A. and Wu, J.J. (2008) "Advances in collagen cross-link analysis. Methods, 45, 65-74.
[8] Svensson, L., Aszodi, A., Heinegård, D., Hunziker, E.B., Reinholt, F.P., Fassler, R. and Oldberg, Å. (2002) Cartilage oligomeric matrix proteindeficient mice have normal skeletal development. Molecular and Cellular Biology, 22, 4366-4371.

[9] Danielson, K.G., Baribault, H., Holmes, D.F., Graham, H., Kadler, K.E. and Iozzo, R. (1997) Targeted disruption of decorin leads to abnormal collagen fibril morphology and skin fragility. Journal of Cell Biology, 136, 729-743.

[10] Svensson, L., Aszodi, A., Reinholt, F.P., Fassler, R., Heinegård, D. and Oldberg, A. (1999) Fibromodulin-null mice have abnormal collagen fibrils, tissue organization, and altered lumican deposition in tendon. Journal of Biological Chemistry, 274, 9636-9647.

[11] Chakravarti, S., Petroll, W.M., Hassell, J.R., Jester, J.V., Lass, J.H., Paul, J. and Birk, D.E. (2000) Corneal opacity in lumican-null mice: defects in collagen fibril structure and packing in the posterior stroma. Investigative Ophthalmology and Visual Science, 41, 3365-3373.

[12] Schonherr, E., Witsch-Prehm, P., Harrach, B., Robenek, H., Rauterberg, J. and Kresse, H. (1995) Interaction of biglycan with type I collagen. Journal of Biological Chemistry, 270, 2776-2783.

[13] Mao, J.R., Taylor, G., Dean, W.B., Wagner, D.R., Afzal, V., Lotz, J.C., Rubin, E.M. and Bristow, J. (2002) Tenascin-X deficiency mimics Ehlers-Danlos syndrome in mice through alteration of collagen deposition. Nature Genetics, 30, 421-425.

[14] Kvist, A.J., Johnson, A.E., Mörgelin, M., Gustafsson, E., Bengtsson, E., Lindblom, K., Aszodi, A., Fassler, R., Sasaki, T. and Timpl, R. (2006) Chondroitin sulfate perlecan enhances collagen fibril formation. Implications for perlecan chondrodysplasias. Journal of Biological Chemistry, 281, 33127-33139.

[15] Wiberg, C., Klatt, A.R., Wagener, R., Paulsson, M., Bateman, J.F., Heinegård, D. and Mörgelin, M. (2003) Complexes of matrilin-1 and biglycan or decorin connect collagen VI microfibrils to both collagen II and aggrecan Journal of Biological Chemistry, 278, 37698-37704.

[16] Bornstein, P., Kyriakides, T.R., Yang, Z., Armstrong, L.C. and Birk, D.E. (2000) Thrombospondin 2 modulates collagen fibrillogenesis and angiogenesis. Journal of Investigative Dermatology, 5, 61-66.

[17] Ruggeri, A., Benazzo, F. and Reale, E. (1979) Collagen fibrils with straight and helicoidal microfibrils: a freeze-fracture and thin section study. Journal of Ultrastructure Research, 68, 101-108.

[18] Reale, E., Benazzo, F. and Ruggeri, A. (1989) Differences in the microfibrillar arrangement of collagen fibrils. Distribution and possible significance. Journal of Submicroscopic Cytology, 13, 135-143.

[19] Raspanti, M., Ottani, V. and Ruggeri, A. (1989) Different architectures of the collagen fibril: morphological aspects and functional implications. International Journal of Biological Macromolecules, 11, 367-371.

[20] Birk, D.E. and Mayne, R. (1997) Localization of collagen types I, III and V during tendon development. Changes in collagen types I and III are correlated with changes in fibril diameter. European Journal of Cell Biology, 72, 352-361.

[21] Raspanti, M., Viola, M., Sonaggere, M., Tira, M.E. and Tenni, R. (2007) Collagen fibril structure is affected by 
collagen concentration and decorin. Biomacromolecules, 8, 2087-2091.

[22] Smith, J.W. (1968) Molecular pattern in native collagen. Nature, 219, 157-158.

[23] Holmes, D.F., Gilpin, C.J., Baldock, C., Ziese, U., Koster, A.J. and Kadler, K.E. (2001) Corneal collagen fibril structure in three dimensions: Structural insights into fibri1l assembly, mechanical properties, and tissue organization. Proceedings of the National Academy of Science U.S.A., 98, 7307-7312.

[24] Cameron, G.J., Alberts, I.L., Laing, J.H. and Wess, T.J. (2002) Structure of Type I and Type III heterotypic collagen fibrils: An X-ray diffraction study. Journal of Structural Biology, 137, 15-22.

[25] Marchini, M., Morocutti, M., Ruggeri, A., Koch, M.H.J., Bigi, A. and Roveri, N. (1986) Differences in the fibril structure of corneal and tendon collagen. An electron microscopy and X-ray diffraction investigation. Connective Tissue Research, 15, 269-281.
[26] Mechanic, G.L., Katz, E.P., Henmi, M., Noyes, C. and Yamauchi, M. (1987) Locus of a histidine-based, stable trifunctional, helix to helix collagen cross-link: stereospecific structure of type I skin fibrils. Biochemistry, 26, 3500-3509.

[27] Yamamoto, S., Hashizume, H., Hitomi, J., Shigeno, M., Sawaguchi, S., Abe, H. and Ushiki, T. (2002) The subfibrillar arrangement of corneal and scleral collagen fibrils as revealed by scanning electron and atomic force microscopy. Archives of Histology and Cytology, 63, 127-135.

[28] Birk, D.E., Fitch, J.M., Babiarz, J.P., Doane, K.J. and Linsenmayer, T.F. (1990) Collagen fibrillogenesis in vitro: interaction of types I and V collagen regulates fibril diameter. Journal of Cell Science, 95, 649-657.

[29] Wenstrup, R.J., Florer, J.B., Brunskill, E.W., Bell, S.M., Chervoneva, I. and Birk, D.E. (2004) Type V collagen controls the initiation of collagen fibril assembly. Journal of Biological Chemistry, 279, 53331-53337. 